\section{AB0561 B CELL THERAPY IN REFRACTORY/ RELAPSED ANCA ASSOCIATED VASCULITIS- A SINGLE CENTRE PROSPECTIVE OBSERVATIONAL STUDY}

G.G. Ekbote, R. Gupta, N. Mendiratta, N. Negalur, M. Bindroo, V. Singal. Rheumatology, MEDANTA, the Medicity, Gurgaon, India

Background: Rituximab (Rtx), a novel biological, having B cell depletion mechanism is an anti- CD 20 antibody and is found to be useful in patients of ANCA associated vasculitis. In AAV the disease activity correlates with increased circulating B cells. Rituximab has been found to be useful in depleting these $B$ cells. According to the RAVE study, Rituximab was shown to be non-inferior to Cyclophosphamide in inducing remission. It also showed that the regimen (Rtx) may be superior to the standard regimen of Cyclophosphamide and glucocorticoids for remission induction in severe relapsing ANCA-associated vasculitis.

In our study, B cell therapy was given in those patients only who had persistent disease activity or relapse.

Objectives: To assess response of Rtx in relapsed/refractory cases of AAV and show that it is a good therapeutic stratergy in such cases.

Methods: In our cohort there were 49 patients of ANCA associated vasculitis, diagnosed by clinical and serological criteria, (by both ELISA and IFA) classified according to ACR criteria and supported, wherever possible, by biopsy. In this prospective study, patients were seen during January 2012 to January 2017 A total of 15 patients received Rituximab for various reasons. Rituximab (Rtx) was given as 1 gram infusion on day 1 and day 15 as induction therapy and subsequently 6 monthly maintenance doses of $500 \mathrm{mg}$ were administered. No other immunosuppression other than steroids were given.

Results: Median follow up was 22 months. All patients had recieved Cyclophosphamide (median dose 6 grams) and $1 \mathrm{mg} / \mathrm{kg}$ glucorticoids at onset. Among the patients who received Rituximab, all had anti PR3 antibody positive \& all were GPA clinically. 14 patients (93.33\%) had lung involvement, renal involvement was seen in $7(46.6 \%)$ patients, $13(86.6 \%)$ patients had upper respiratory tract involvement, $6(40 \%)$ had ophthalmic involvement. Nervous system involvement was seen in $5(33.3 \%)$ and myocarditis was seen in $3(20 \%)$ each. $3(20 \%)$ patients had gangrene.

Indications for receiving Rtx were heterogenous. It was given for involvement of lung, renal, ophthalmic, upper respiratory and nervous system in $6(40 \%), 3$ $(20 \%), 3(20 \%), 1(6.66 \%)$ and $1(6.66 \%)$ respectively. Whereas $1(6.66 \%)$ patient received Rtx for persistent disease activity.

12 out of 15 patients $(80 \%)$ achieved remission at mean follow up of 3 months while one achieved at 6 months follow up $\&$ all maintained continued remission. 1 patient was due for 3-month follow up. 1 patient died due to lung infection during the course. 4 patients had permanent morbidities/organ damage which they already had before starting Rtx. Only one patient had infusion reaction at the end of 1 st induction however she remained in remission after the first dose itself. Conclusions: $86.6 \%$ patients achieved remission after Rtx and remained in continous remission at median follow up of 22 months. Rtx is a very good therapeutic strategy for refractory/relapsed especially PR3+ AAV also it can be used as a maintenance regimen for long term.

References:

[1] Stone JH, Merkel PA, Spiera R, Seo et al. Rituximab versus cyclophosphamide for ANCA-associated vasculitis. N Engl J Med. 2010;363:221-23.

Disclosure of Interest: None declared

DOI: 10.1136/annrheumdis-2017-eular.5596

\section{AB0562 CLASSIFICATION OF ANCA ASSOCIATED VASCULITIS BASED ON PR3 AND MPO SEROLOGY \& THEIR OUTCOME: A SINGLE CENTRE PROSPECTIVE STUDY}

G.G. Ekbote, R. Gupta, N. Mendiratta, N. Negalur, M. Bindroo, V. Singal.

Rheumatology, MEDANTA, the Medicity, Gurgaon, India

Background: It is often difficult to classify small vessel vasculitis, especially AAV, as Granulomatosis polyangitis [GPA], Microscopic polyangitis [MPA], Eosinophilic granulomatosis polyangitis [EGPA] \& Idiopathic cresentic glomerulonephritis. But with the discovery of ANCA, rheumatologists divide this as ANCA positive or negative vasculitis.

Objectives: To classify AAV as anti-proteinase 3 (PR3) antibody+ or antimyeloperoxidase (MPO) antibody $+\&$ compare their clinical presentation \& outcome.

Methods: 49 patients were included in our study from August 2011 till January 2017. Patients were classified according to PR3 and MPO serology [based on ELISA].

Results: Median follow up was 18 months. PR3 + were 38 and 11 patients were MPO+. GPA was significantly higher in PR3 Group vs MPO group [36 (94.7\%) vs $1(9.1 \%) p<0.0001^{*}$ ] while MPA was significantly lower in PR3 group as compared to MPO (2 [5.3\%] vs $\left.5[45.45 \%] p=0.001^{\star}\right)$. All EGPAs were MPO+ $(4[36.35 \%])$. 48 fulfilled ACR clinical criteria for GPA/MPA or EGPA. 1 patient with arteritic anterior ischemic optic neuropathy without any other major organ involvement had significantly higher titres of MPO antibodies \& was sorted as unclassified AAV. None were idiopathic crescentic glomerulonephritis in our cohort. 18 were biopsy proven [15 PR3+vs $3 \mathrm{MPO}+$ ]. Lung involvement was significantly higher in PR3 group than MPO group (32 [84.2\%] vs $\left.6[54.5 \%] p=0.037^{\star}\right)$.Kidney involvement was also more in PR3 group but was not statistically significant (20 [52.6\%] vs 4 [36.4\%] $p=0.341)$. Upper respiratory involvement was significantly higher in PR3 group $\left(26[68.4 \%]\right.$ vs $\left.3[27.3 \%] p=0.014^{*}\right)$.

Comparision between manifestations of ophthalmic, cardiac, peripheral vascular system \& nervous systems of PR3+ \& MPO+ groups was not statistically significant.

Complete remission without permanent organ damage was seen in $16(42 \%)$ vs $6(54.5 \%)$ in PR3 and MPO groups respectively $(p=0.465)$. Frequency of relapse/refractory disease, though higher in PR3 group, was not statistically significant (PR3 vs MPO, 10 [26.3\%] vs 1 [9.1\%] $\mathrm{p}=0.228$ ). Rates of morbidity \& mortality were not significant statistically between PR3 \& MPO groups (11 [28.9\%] vs $2[18.2 \%] p=0.476 \& 3[7.9 \%]$ vs $1[9.1 \%] p=0.899$ respectively). Similar comparisons were made between those who were classified clinically as GPA, MPA \& EGPA with respect to remission, relapse, morbidity and mortality. All EGPAs achieved remission. Comparison between groups when divided as GPA \& PR3 and MPA \& MPO did not show statistical significance. 15 patients (all clinically GPA \& PR3+) of the cohort [39.5\%] received rituximab for relapse/refractory disease during/after initial induction therapy with cyclophosphamide \& steroids. Conclusions: In this study, we did not find any advantage of clinical classification over serological. Wrongly diagnosing patients when disease is still evolving \& inter-clinician bias are eliminated when classifying patients according to serology. Classification as PR3 \& MPO is simpler and universal.

\section{References:}

[1] Hunder, G. G. et al. The American College of Rheumatology 1990 criteria for the classification of vasculitis. Introduction. Arthritis Rheum. 33,1065-1067 (1990).

[2] Houben E, Bax WA, van Dam B, et al.Diagnosing ANCA-associated vasculitis in ANCA positive patients: A retrospective analysis on the role of clinical symptoms and the ANCA titre. Carubbi. F, ed. Medicine. 2016;95(40):e5096.

Disclosure of Interest: None declared

DOI: 10.1136/annrheumdis-2017-eular.4878

\section{AB0563 RITUXIMAB IN PATIENTS WITH TAKAYASU ARTERITIS: A SEVEN PATIENTS EXPERIENCE}

G. Pazzola ${ }^{1}$, F. Muratore ${ }^{1}$, N. Pipitone ${ }^{1}$, F. Crescentini ${ }^{1}$, P. Cacoub ${ }^{2}$ L. Boiardi ${ }^{1}$, L. Spaggiari ${ }^{3}$, C. Comarmond ${ }^{2}$, S. Croci ${ }^{4}$, D. Saadoun ${ }^{2}$,

C. Salvarani ${ }^{1}{ }^{1}$ Rheumatology, Arcispedale S Maria Nuova, Reggio Emilia, Italy; 2 Internal Medicine and Clinical Immunology, Groupe Hospitalier PitieSalpetriere, Paris, France; ${ }^{3}$ Radiology; ${ }^{4}$ Inflammation Laboratory, Clinical Immunology, Allergology and Advanced Biotechnologies Unit, Arcispedale S Maria Nuova, Reggio Emilia, Italy

Background: Takayasu arteritis (TAK) is a large vessel vasculitis involving the aorta and its major branches in patients younger than 40 years. Glucocorticoids (GCs) are the mainstay of treatment for TAK, but relapses and GC dependence are seen in more than two-thirds of patients. Increasing evidence supports a role for $B$ cells in the pathogenesis of TAK. Circulating plasmablasts and memory $B$ cells are increased, while naive $B$ cells are decreased in patients with active TAK as compared with inactive and control patients [1]. These findings suggest a potential role for B cell depleting therapy in TAK.

Objectives: Our aim was to assess the efficacy and safety of Rituximab (RTX) in a series of 7 patients with TAK.

Methods: We conducted an open-label study on 7 TAK patients (5 followed prospectively, 2 retrospective cases) treated with RTX. All patients satisfied the American college of Rheumatology classification criteria for TAK. Six of the 7 patients had a disease refractory to high dose GCs and conventional immunosuppressive (IS) and/or biologic agents. One newly diagnosed, treatment naïve TAK patient refused GCs and received RTX alone. RTX was administered according to rheumatoid arthritis scheme (2 infusions of $1.000 \mathrm{mg}, 15$ days apart). Clinical evaluation, laboratory tests (full blood count, ESR, CRP) and imaging modalities (CTA or MRA, and PET/CT) were performed at first RTX administration and every 6 months thereafter. Disease activity was assessed using Kerr index. Radiographic disease progression was defined as new or worsening lesions at follow-up CTA or MRA. PET/CT was considered positive for active disease if two or more large vessels showed grade 2 FDG uptake or higher.

Results: Seven patients ( 6 females) were included in the study. Mean (SD) age was $32.4( \pm 16.1)$ years. At first RTX administration, all patients had active disease

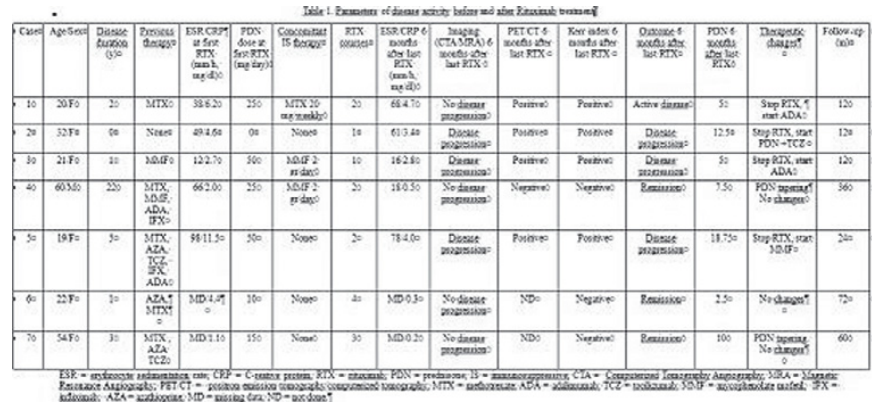


according to Kerr index ( $>2$ ), and had also evidence of active disease at PET/CT. Table 1 summarizes the main results of our study.

Despite RTX treatment, 4 of the 7 patients had evidence of persistent disease activity and/or radiographic disease progression during follow-up.

Three out of 7 patients in whom RTX was employed as rescue therapy achieved complete remission.

Conclusions: Our data do not support a role for RTX as first line biologic therapy in TAK patients, but it may have a role in some patients as second/third line biological therapy.

References:

[1] Hoyer BF et al. Takayasu arteritis is characterised by disturbances of B cell homeostasis and responds to B cell depletion therapy with rituximab. Ann Rheum Dis 2012;71:75-9. doi:10.1136/ard.2011.153007.

Disclosure of Interest: None declared

DOI: 10.1136/annrheumdis-2017-eular.5158

\section{AB0564 TEMPORAL ARTERITIS: IS THERE ANY CORRELATION BETWEEN ULTRASONOGRAPHIC ARTERIAL WALL INVOLVEMENT AND THE INFLAMMATORY CELLULAR INFILTRATE AT HISTOLOGICAL EXAMINATION?}

G. Germano ${ }^{1}$, P.L. Macchioni ${ }^{1}$, A. Cavazza ${ }^{2}$, L. Cimino $^{3}$, F. Muratore ${ }^{1}$, C. Salvarani ${ }^{1} .{ }^{1}$ Rheumatology Unit; ${ }^{2}$ Pathology Unit; ${ }^{3}$ Ophthalmology Unit, IRCCS Arcispedale Reggio Emilia, Reggio Emilia, Italy

Background: Ultrasonographic alterations such as the halo sign and the compression test are now accepted as surrogate markers of artery inflammation. No data have yet been published on the correlation between the ultrasonographic grading of arterial wall inflammation and the grading of cellular infiltrate.

Objectives: To compare a semiquantitative ultrasonographic grading (USG) of TA involvement (halo sign and media-intima thickness) with a semiquantitative grading of the inflammatory burden in patients with giant cell (temporal) arteritis (GCA).

Methods: Fiftheen consecutive patients with new onset clinical symptoms and satisfiing ACR criteria for GCA, with positive halo sign in the frontal branch and positive temporal artery biopsy have been enrolled. For each patients we performed power Doppler ultrasonography of temporal artery with a $18-6 \mathrm{MHz}$ linear probe (Esaote MyLab 70) and measured the maximum halo thickness of TA frontal branch in a quantitative and semiquantitave (0-3) grade of involvement $(0=<0.37 \mathrm{~mm}, 1=$ in between $0.38-0.44,2=0.45-0.6 \mathrm{~mm}, 3=>0.6)$. TA biopsy was done in the same frontal branch evalutaed with US. Then we compared the ultrasonographic data with a semiquantitative (0: absent, 1: mild, 2: moderate, 3: severe) grading of the trasmural cellular inflammatory infiltrate and with the intima-media thickness of the TA biopsy specimen. Moreover US results were correlated with the other patterns of histological alterations (giant cells, calcifications, laminar necrosis). Correlation between variables was done by Rho of Spearman method.

Ultrasonography of temporal artery: grading of Halo thickness

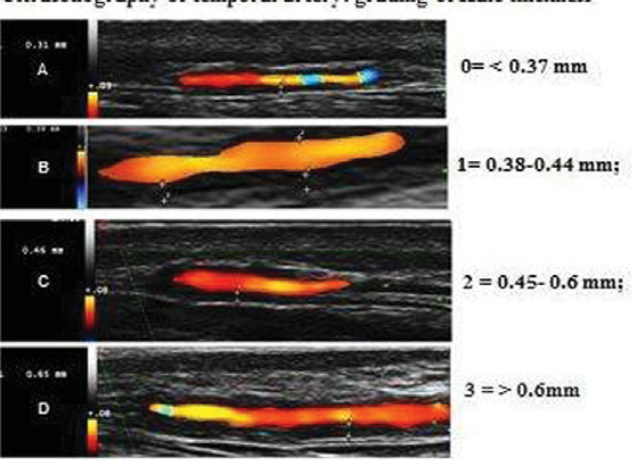

Grading of the trasmural cellular inflammatory infiltrate and intimamedia thickness of the TA biopsy.

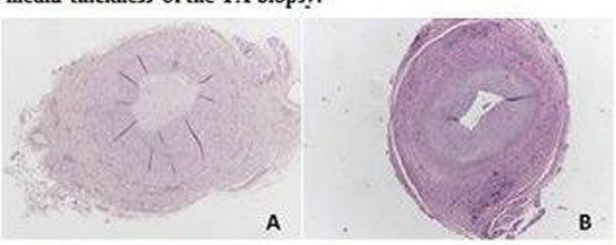

A) Mild inflammation with mild intimal hyperplasia;

B) Moderate inflammation with moderate intimal hyperplasia;

C) Severe inflammation with severe intimal hyperplasia;
Results: 15 patients, 6 males and 9 females (mean age $71.6 \pm 7$ years - duration symptoms at onset $1.7 \pm 1.3$ months - mean ESR $60 \mathrm{~mm} / \mathrm{h} \pm 29-$ mean CRP 8 $\mathrm{mg} / \mathrm{dl} \pm 5.2)$ entered the study. US halo sign was bilateral in $10 / 15(66,7 \%)$. The mean halo thickness was $0.53 \mathrm{~mm} \pm 0.12$. Five patients had $U S G=1$, six patients $=2$ and four patients $=3$. The hystological inflammatory grade 1 was present in seven pts, grade 2 in four and grade 3 in four pts. No significant correlation were found between USG and histological inflammatory grade, nor with the presence of giant cells, calcifications, laminar necrosis and intima-media thickness.

Conclusions: No correlation has been found between the size of the halo sign and the hystological inflammatory grading.

Disclosure of Interest: None declared

DOI: 10.1136/annrheumdis-2017-eular.5093

\section{AB0565 INSUFFICIENT INFLUENZA VACCINATION COVERAGE IN GIANT CELL ARTERITIS. A FRENCH POPULATION-BASED STUDY}

G. Pugnet ${ }^{1}$, M. Lapeyre-Mestre ${ }^{2}$, M. Mounie ${ }^{2}$, G. Moulis ${ }^{1}$, L. Sailler ${ }^{1}$. ${ }^{1}$ Service de Médecine Interne, CHU Toulouse Purpan; ${ }^{2}$ INSERM UMR 1027, Toulouse, France

Background: French general health insurance scheme has implemented a national influenza vaccination program, including full refund for those 65 years old or more. Despite this program, only 62.6 to $71 \%$ of people aged over 65 years are vaccinated and no data are available regarding influenza vaccination coverage (IVC) in GCA.

Objectives: To evaluate the rate and the factors associated with seasonal influenza vaccination coverage in incident giant cell arteritis (GCA) patients compared with controls.

Methods: Using the French National Health Insurance system (SNIIRAM), we included patients with incident GCA from the Midi-Pyrenees region, southern France, and randomly selected 6 controls matched by sex and age at calendar date from January 2005 to December 2008 and followed them till April 2011. The vaccination rate was estimated from vaccine dispensation registered in the SNIIRAM. IVC was compared between GCA and their controls using longitudinal multivariate Poisson regression.

Results: Eighty-seven incident GCA patients over 65 years of age and 509 in the control group were included. Mean follow-up was $4.4 \pm 1.2$ and $4.2 \pm 1.2$ years, respectively. During the yearly influenza campaigns from 2005-2006 to 2010-2011, the IVC rates in the GCA group and the control group ranged from 60.8 to $74.7 \%$ vs 56.6 to $70.4 \%$, respectively. Incident GCA influenza vaccination rate was $20 \%$ higher than controls $(R R=1.20$; IC 1.09 to $1.32, P<0.001)$ (Table 1).

Table 1. Associated factors with recommended influenza vaccination (Multivariate longitudinal Poisson analysis)

\begin{tabular}{|c|c|c|c|c|}
\hline & & Adjusted RR & IC 95\% & $P$ \\
\hline$\overline{G C A}$ & GCA & 1.20 & $1.09-1.32$ & $<0.001$ \\
\hline \multicolumn{5}{|l|}{ Comorbidity at baseline } \\
\hline - High blood pressure & & 1.13 & $1.04-1.24$ & 0.006 \\
\hline \multirow[t]{3}{*}{ Age, years } & $164.4 ; 71.5]$ & 1 & & \\
\hline & $171.5 ; 80]$ & 1.15 & $1-1.32$ & 0.048 \\
\hline & $180 ; 91.9]$ & 1.13 & $0.99-1.29$ & 0.079 \\
\hline Gender & Eemale & 1.05 & $0.96-1.16$ & 0.259 \\
\hline \multirow[t]{3}{*}{ Time, Influenza camoaigns. } & [2005: 2008] & 1 & & \\
\hline & [2008: 2010] & 1.11 & $1.08-1.14$ & $<0.001$ \\
\hline & [2010 : 2011] & 1.05 & $1.02-1.08$ & 0.002 \\
\hline \multirow[t]{2}{*}{ GCA"Time } & $\mathrm{GCA}^{*}[2008: 2010]$ & 0.90 & $0.87-0.92$ & $<0.001$ \\
\hline & $G^{\prime} *^{*}[2010: 2011]$ & 0.77 & $0.72-0.83$ & $<0.001$ \\
\hline
\end{tabular}

Conclusions: Rates of recommended influenza vaccinations are insufficient in GCA patients over 65 years. More attention should be paid for appropriate vaccination during GCA course.

Disclosure of Interest: None declared

DOI: 10.1136/annrheumdis-2017-eular.1814

\section{AB0566 18F-FDG-PET-POSITIVE TEMPORAL ARTERY IN GIANT CELL ARTERITIS: A POSSIBLE CORRELATION WITH HISTOPATHOLOGICAL FINDINGS}

H. Kono ${ }^{1}$, Y. Okada ${ }^{1}$, K. Asako ${ }^{1}$, H. Kikuchi ${ }^{1}$, Y. Oguchi ${ }^{1}$, Z.-I. Honda ${ }^{1}$

A. Takeuchi ${ }^{1}$, T. Tokairin ${ }^{2}$, K. Saito ${ }^{2} .{ }^{1}$ Department of Internal Medicine;

${ }^{2}$ Department of Pathology, Teikyo University School of Medicine, Tokyo, Japan

Background: Giant cell arteritis (GCA) a form of vasculitis in persons older than 50 years, in which cranial and systemic large vessels can be involved. ${ }^{18} \mathrm{~F}$-fluorodeoxyglucose positron emission tomography - computed tomography $\left({ }^{18} \mathrm{~F}\right.$-FDG-PET-CT) is increasingly used to diagnose inflammation of the large arteries in GCA. But it is very rare to observe an inflammation of temporal arteries of GCA using ${ }^{18}$ F-FDG-PET. 